\title{
Capacitação em gerenciamento do risco de inundações e movimentos gravitacionais de massa para profissionais de defesa civil
}

\author{
TRAINING IN RISK MANAGEMENT OF FLOOD AND GRAVITATIONAL MASS MOVEMENT FOR CIVIL DEFENSE PROFESSIONALS
}

\author{
Silvia Midori Saito ${ }^{1}$, Andrea Fregolente Lazaretti ${ }^{2}$, Eduardo Cesar Fernandes Filho ${ }^{3}$, Carla Corrêa Prieto ${ }^{4}$, Gabriel Guimaräes Facuri ${ }^{5}$, Gilberto Lima ${ }^{6}$, \\ Renato Santos Lacerda? \\ 1 - Pesquisadora, Centro Nacional de Monitoramento e Alertas de Desastres Naturais, São José dos Campos, SP. Programa de Pós-Graduação em Desastres \\ Naturals, Universidade Federal de Santa Catarina, CAMPuS Universitário Trindade, Florianópolis, SC \\ 2 - Pesquisadora em Geociências, Serviço Geológico do Brasil, São Paulo, SP \\ 3 - Capitão PM, Pós-Graduando em Engenharia Geotécnica, Comando de Policiamento Metropoltano, São Paulo, SP \\ 4 - Tecnologista, Centro Nacional de Monitoramento e Alertas de Desastres Naturais (Cemaden), São José dos Campos, SP \\ 5 - Pesquisador em Geociências, Serviço Geológico do Brasil, são Paulo, SP \\ 6 - Analista em Geociências, Serviço Geológico do brasil, São Paulo, SP \\ 7 - Analista em Ciência e Tecnologia, Centro Nacional de Monitoramento e Alertas de Desastres Naturals, São José dos Campos, SP

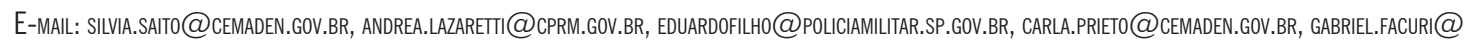 \\ CPRM.GOV.BR, GILBERTO.LIMA@CPRM.GOV.BR, RENATO.LACERDA@CEMADEN.GOV.BR
}

\begin{abstract}
Continuous training is necessary for civil defense professionals to comply their work skills, concerning the broad scope of work for identification and assessment of hazards, susceptibilities and vulnerabilities to disasters. This manuscript presents the experience in the intra and intergovernmental planning and execution of a training course, for an integrated practice for disaster risk management. Three management tools were presented to the participants: sectorization of risk areas for mass movements and floods; monitoring and alerting; and contingency plans. The course consisted of theoretical and practical classes, which highlighted the importance of knowing and monitoring risks in an integrated manner, as well as to improving the disaster response. In total, 74 civil defense technicians from 32 municipalities were trained in two editions of the course. This training encouraged greater autonomy for civil defenses to act locally in disaster risk management.
\end{abstract}

Resumo: Capacitação contínua é necessária para que os profissionais de defesa civil realizem suas ações, em vista do amplo campo envolvido na identificação e avaliação de ameaças, suscetibilidades e vulnerabilidades a desastres. Este texto descreve experiência de planejamento e execução intra e intergovernamental de um curso presencial de capacitação, buscando integrar, de forma prática, 0 gerenciamento de risco de desastres. Três instrumentos de gerenciamento foram apresentados aos participantes: setorização de áreas de risco para movimentos de massa e inundações; monitoramento e alerta; e planos de contingência. 0 curso foi composto de aulas teóricas e práticas, que evidenciaram a importância de conhecer e monitorar os riscos de forma integrada, além de aperfeiçoar a resposta diante da ocorrência de desastres. Em duas edições do curso, foram capacitados 74 técnicos de defesa civil de 32 municípios, o que possibilitou maior autonomia desses profissionais para atuar localmente no gerenciamento do risco de desastres.
Citation/Citação: Saito, S. M., Lazaretti, A. F.,Fernandes Filho, E. C., Prieto, C. C., Facuri, G. G., Lima, G., \& Lacerda, R. S. (2020). Capacitação em gerenciamento do risco de inundações e movimentos gravitacionais de massa para profissionais de defesa civil. Terræ Didatica, 16, 1-12, e020035. doi: 10.20396/td.v16i0.8659129

Keywords: Civil Defense, Risk sectorization, Early warning system, Contingency plan.

Palavras-chave: Defesa Civil, Setorização de risco, Sistema de alerta, Plano de contingência.

Manuscript/Manuscrito:

Received/Recebido: 14/04/2020

Revised/Corrigido: 11/06/2020

Accepted/Aceito: 25/08/2020

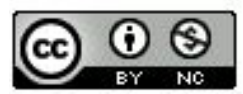

\section{Introdução}

Ao longo dos últimos anos observa-se crescente preocupação em formular estratégias para a redução do risco de desastre $(\mathrm{RRD})^{1}$ baseada na resiliência

1 A UNDRR (2017) acentua que uma política objetiva de Redução do Risco de Desastre (RRD) visa prevenir que novos riscos de desastres surjam e, ao mesmo tempo, atuar para que haja redução dos riscos de desastres existentes, gerenciando riscos residuais e fortalecendo a resiliência. Essa política de redução de comunidades. Em termos de agendas globais, destacam-se: (1) o Marco de Ação de Sendai, do qual o Brasil é signatário, preconizando a redução substancial do risco de desastre, meios de subsistência e saúde, bem como de ativos econômicos, físicos, sociais, culturais, ambientais, de pessoas,

do risco de desastre configura um gerenciamento do risco de desastre, traduzida em estratégias e planos locais, regionais, nacionais e internacional. 
empresas, comunidades e países, para o período de 2015-2030 (United Nations International Strategy for Disaster Reduction [UNISDR], 2015); (2) mais recentemente, a Agenda 2030, por meio dos Objetivos do Desenvolvimento Sustentável (ODS), dispõe sobre uma série de metas para que os países subscritores alcancem melhorias relacionadas a diversos aspectos, como saúde, educação e meio ambiente. O ODS 11, sobre cidades e comunidades sustentáveis, apresenta uma meta relacionada diretamente à redução de danos humanos e materiais causados por desastres ${ }^{2}$.

A temática de RRD está presente nas discussões globais, motivando ações que visem comunidades mais bem preparadas para o risco de desastre, entendido como o potencial de perda de vidas, ferimentos, bens destruídos ou danificados que podem ocorrer a um sistema, sociedade ou comunidade em um período específico de tempo, determinado de modo probabilístico em função de risco, exposição, vulnerabilidade e capacidade (United Nations Office for Disaster Risk Reduction [UNDRR], 2017).

No Brasil, apesar dos esforços governamentais em políticas objetivas na RRD, em especial na última década (Bertone \& Marinho, 2013, Cardona et al., 2017), danos humanos e materiais ainda continuam sendo registrados, comprometendo o desenvolvimento sustentável no país. Um estudo abrangendo a análise de 825 municípios brasileiros afetados por deslizamentos e inundações, estimou que 8.266.566 pessoas estavam expostas em áreas de risco, o que equivale a $9 \mathrm{em}$ cada 100 habitantes (Alvalá et al., 2019). Quanto aos prejuízos econômicos, desastres de grande magnitude ocorridos no Brasil (Vale do Itajaí, 2008; Pernambuco e Alagoas, 2010; região Serrana do Rio de Janeiro, 2011) somaram aproximadamente $R \$ 15,3$ bilhões de prejuízos, dos quais $\mathrm{R} \$$ 9,4 bilhões em custos diretos e $\mathrm{R} \$ 5,9$ bilhões em custos indiretos (Global

20 conceito de desastre é dado como uma perturbação ou ruptura séria do funcionamento de uma comunidade ou sociedade em qualquer escala, devido a eventos ameaçadores que interagem com as condições de exposição, vulnerabilidade e capacidade, levando a perdas e impactos humanos, materiais, econômicos e ambientais (UNDRR, 2017). No presente artigo, "desastre natural" foi empregado seguindo o que está presente em leis, decretos, instruções normativas, tal qual encontrada na Lei 12.608, de 10 de abril de 2012: da Lei 8.036, de 11 de maio de 1990: do Decreto 9.677, de 2 de janeiro de 2019: Instrução Normativa, $n^{\circ} 1$, de 24 de agosto de 2012, Anexo I, do antigo Ministério da Integração Nacional que elaborou a Classificação e Codificação Brasileira de Desastres (COBRADE) a partir da linha classificatória do Banco de Dados Internacional de Desastres (EM-DAT), no qual distingue duas categorias genéricas de desastres (natural e tecnológico).
Facility for Disaster Reduction and Recovery [GFDRR] \& World Bank [WB], 2014). Frente aos números dessa magnitude, nota-se que uma solução definitiva para resolver esse problema ainda está longe de ser alcançada. Assim, a convivência com o risco de desastre, por meio de medidas estruturais e não-estruturais, como planejamento urbano, mapeamento de áreas de risco, sistema de alertas, planos de contingência e educação para redução de risco de desastre, podem ser considerados como meios razoáveis para diminuir os impactos em toda a sociedade (Andjelkovic, 2001; Gruntfest, 2010; Kundzewicz, 2002). Essas medidas fazem parte de um macroprocesso, aqui entendido como gestão de riscos de desastres, que "(...) compreende o planejamento, a coordenação e a execução de ações e medidas preventivas destinadas a reduzir os riscos de desastre e evitar a instalação de novos riscos" (Ministério da Integração Nacional, 2017, p.24).

A gestão de risco de desastre no Brasil ainda constitui um grande desafio para as instituições que compõem o Sistema Nacional de Proteção e Defesa Civil (Di Gregorio et al., 2013). Os autores destacam alguns pontos cruciais para a reformulação, como a integração de atividades e processos, uniformização de procedimentos e protocolos, melhoria do fluxo de informações e comunicações entre as instituições. O intercâmbio de informações entre as diferentes instituições é fundamental para o gerenciamento do risco de desastre (Kobiyama et al., 2004).

No intuito de contribuir com a política nacional de redução do risco de desastre, o presente manuscrito tem como objetivo apresentar a experiência intra e intergovernamental que envolveu órgãos/ entidades da União e do Estado no planejamento e execução de um curso de capacitação integrado para profissionais de defesa civil dos entes federativos municipais do Estado de São Paulo. O arranjo de cooperação institucional envolveu o Serviço Geológico do Brasil (SGB-CPRM), o Centro Nacional de Monitoramento e Alertas de Desastres Naturais (Cemaden) e a Coordenadoria Estadual de Proteção e Defesa Civil de São Paulo (Cepdec-SP). A escolha pelo público alvo deste curso se fundamentou no fato de os profissionais de defesa civil serem responsáveis, em maior ou menor grau, pelas ações de prevenção, resposta e recuperação em desastres em seus municípios. Ao contrário do que prega o senso comum, o campo de ação da defesa civil vai além do socorro pós-desastre, mas também pós-impacto, cabendo ainda articular e mobilizar meios logísticos, estimular ações preventivas e de preparação da população (Séguin, 2012). 
Diante do amplo do campo de ação, treinamentos e capacitações se fazem necessários para a formação técnica dos profissionais de defesa civil (Alexander et al., 2009). Um diagnóstico sobre a necessidade de formação para técnicos de defesa civil no Brasil evidenciou que as maiores limitações estavam relacionadas às dificuldades em gerir o risco de desastre existente, de realizar articulação interinstitucional e de condições restritivas para desempenho de suas funções organizacionais (Programa das Nações Unidas para o Desenvolvimento [PNUD], 2014). Esses técnicos comumente desempenham outras atividades relacionadas às secretarias ou departamentos a que estão vinculados, como meio ambiente, trânsito ou guarda municipal (Londe et al., 2015). Além disso, muitos desses profissionais desenvolveram suas habilidades na prática cotidiana, o que evidencia a necessidade de construção de competências e capacitação teórica conceitual associada à prática de campo (Monteiro \& Brito Junior, 2017). Nesse contexto, a sistematização de conhecimento sobre as práticas em proteção e defesa civil se mostra cada vez mais necessária.

As instituições envolvidas na organização do curso têm em comum a atuação próxima aos técnicos de defesa civil municipal, os quais são usuários diretos dos seguinte produtos: (1) das setorizações de risco de movimentos gravitacionais de massa deslizamentos, queda, tombamento, rolamento de blocos de rochas e corridas, e de inundações bruscas e graduais representadas por mapeamentos de risco que são produzidos pelo SGB-CPRM; (2) dos alertas risco de desastres, da previsão do risco geo-hidrológico e de estudos sobre vulnerabilidades, suscetibilidades a desastres e de monitoramento de eventos geológicos e hidrometeorológicos, produzidos pelo Cemaden; (3) e das orientações para elaboração de planos de contingência providas pela Cepdec-SP em consonância com o Cenad (Centro Nacional de Gerenciamento de Riscos e Desastres).

O SGB-CPRM é uma empresa pública, de mais de 50 anos, vinculada ao Ministério de Minas e Energia do governo federal, cuja missão é gerar e disseminar conhecimento geocientífico com excelência, contribuindo para melhoria da qualidade de vida e desenvolvimento sustentável do Brasil (SGB-CPRM, 2020). Uma das maneiras de apoiar o cumprimento da missão da empresa é por meio da expertise de seu corpo técnico em gerar produtos que atendam as demandas da sociedade, frente a questionamentos e situações onde a presença do geocientista se faz necessária. Um dos produtos desenvolvidos no Departamento de Gestão Terri- torial compreende a setorização do risco de movimentos gravitacionais de massa e de inundações, que desde 2011 atendeu mais de 1.605 municípios em todo território brasileiro, além de 180 revisitas, totalizando $1785^{3}$ municípios.

O Cemaden, por sua vez, é uma unidade de pesquisa do Ministério de Ciência, Tecnologia, Inovações. A missão do Centro é desenvolver e disseminar conhecimentos científico-tecnológicos e realizar o monitoramento e a emissão de alertas para subsidiar a gestão do risco de desastre natural e de seus respectivos impactos (Cemaden, 2020). Equipes multidisciplinares, formadas por especialistas em Geociências, Hidrologia, Meteorologia e Vulnerabilidade a Desastres, monitoram 24 horas por dia, 7 dias por semana e emitem alertas de risco de desastres para 958 município brasileiros afetados por deslizamentos, inundações e enxurradas. Os alertas de risco são encaminhados ao Cenad, que sua por vez os redirecionam às coordenações municipais e estaduais de defesa civil. O Cemaden conta também com uma equipe de pesquisadores, responsável pelo desenvolvimento de pesquisa científica, tecnológica e de inovação de forma multidisciplinar, visando à redução de desastres e seus impactos.

A Defesa Civil do Estado de São Paulo foi constituída em razão dos resultados catastróficos decorrentes das chuvas intensas ocorridas em Caraguatatuba em 1967 e dos incêndios dos edifícios Andraus e Joelma em 1972 e 1974, respectivamente, que ocasionaram 203 vítimas e mais de 650 pessoas feridas (Cepdec-SP, 2020). A subsequente análise aos eventos indicou que estes se deram em consequência da falta de coordenação dos órgãos públicos, rápida resposta e ausência de integração com a sociedade civil. Diante da necessidade de criar um órgão central voltado à prevenção e minimização dos danos ocasionados por desastres, tanto naturais como antropogênicos, foi criado, em 9 de fevereiro de 1976, pelo Decreto 7.550, o Sistema Estadual de Defesa Civil, posteriormente reorganizado em 16 de junho de 1995. Este Sistema dispõe de uma Coordenadoria Estadual de Proteção e Defesa Civil, subordinada diretamente ao governador do Estado e dirigida pelo coordenador estadual de Proteção e Defesa Civil. Constitui-se no órgão central do Sistema de Proteção e Defesa Civil do Estado de São Paulo.

3 Dados atualizados em 17/07/2020 - URL: http://www.SGB.gov.br/publique/Gestao-Territorial/ Prevencao-de-Desastres/Produtos-por-Estado--Setorizacao-de-Risco-Geologico-5390.html 
As instituições envolvidas no planejamento e execução do curso de capacitação em gerenciamento do risco de desastre para a Defesa Civil do Estado de São Paulo já tinham entre si estabelecido acordo e protocolo de cooperação. O Cemaden, por exemplo, já havia formalmente consignado uma parceria bilateral com a Casa Militar e outra com a SGB-CPRM e, esta, por sua vez, com a Defesa Civil do Estado de São Paulo. Em que pese um arranjo formal constituído, haveria de ser elaborado um projeto de curso de capacitação interdisciplinar voltado para as coordenações municipais de defesa s civil do estado. Arranjos cooperativos intra-governamental (cooperação entre órgãos e entidades do mesmo ente federativo) e intergovernamental (entre órgãos e entidades de diferentes entes federativos) são considerados ideais para a implementação de planos, estratégias, projetos, ações, atividades de interesse público. A combinação de organizações profissionalizadas com mecanismos efetivos de coordenação intra e intergovernamentais elevam as capacidades técnico-administrativas (Pires \& Gomide, 2016)

Espera-se que a experiência intra e intergovernamental de oferta integrada de capacitação, descrita a seguir, possa influir de alguma forma em aprimorar ou criar novos métodos, abordagens e/ ou práticas de disseminação do conhecimento em gerenciamento do risco de desastre, a fim de que os municípios possam, sob a Lei 12.608/2012, que institui a Política Nacional de Proteção e Defesa Civil (Brasil, 2012), adotar e implementar medidas necessárias à redução do risco de desastre em seus territórios e assim contribuir com a salvaguarda não só de ativos e bens materiais e ambientais, mas principalmente de vidas humanas.

\section{Concepção do curso}

Para a United Nations Office for Disaster Risk Reduction (UNDRR, 2020), a gestão de risco de desastres (GRD) é a aplicação de políticas e estratégias de redução do risco de desastre para evitar novos riscos, reduzir o risco existente e gerenciar riscos residuais, contribuindo para o fortalecimento da resiliência e a redução de perdas de desastres. Trata-se, portanto, de uma abordagem prévia ao desastre, que envolve articulação intersetorial e de políticas públicas, desde níveis de Organizações não Governamentais - ONGs, sociedade civil e universidades (Shaw, 2012). Diante da materialização do desastre, esforços são despendidos para resposta e pronto restabelecimento dos sistemas sociais afetados. Estas ações correspondem ao gerenciamento de desastre, que compreende o planejamento, a coordenação e a execução em resposta e de recuperação (Centro Universitário de Pesquisa e Estudos sobre Desastres [Ceped], 2014); tem-se como exemplo, as medidas emergenciais de socorro e aquelas relacionadas ao retorno das atividades sociais e econômicas das comunidades.

O gerenciamento de risco, por sua vez, está inserido na GRD e operacionaliza uma de suas fases. As etapas do gerenciamento de risco partem de quatro questões básicas e de extrema importância (SGB-CPRM, 2016). A primeira questão é sobre qual o processo que será identificado/mapeado. Tão logo tenham sido definidos os processos, a segunda pergunta é como eles ocorrem, buscando identificar os condicionantes naturais e antrópicos. A terceira pergunta é onde estes eventos ocorrem e por meio de correlações e monitoramento, definir os momentos de maior probabilidade de ocorrência e, por consequência, quais ações estruturais e não-estruturais devem ser tomadas e finalmente quem deve executar cada etapa deste gerenciamento (Figura 1). Vale reforçar que para a definição de todas essas questões existem dois pilares fundamentais que as norteiam: a previsão e a prevenção. O primeiro tratando da espacialização e definição e o segundo ligado as medidas a serem adotadas, visando a redução do impacto econômico e social de um evento (UNDRR, 2017).

Historicamente, algumas entidades públicas brasileiras atuavam de forma pontual nesta temática. A partir de 2003, com a criação do então Ministério das Cidades foi instituída a Ação de Apoio à Prevenção e Erradicação de Riscos em Assentamentos Precários. Tratava de um conjunto de ações para a redução risco nas áreas urbanas, em associação ao Sistema Nacional de Defesa Civil (Carvalho \& Galvão, 2006). Além disso, treinamentos para técnicos e gestores municipais, bem como seminários nacionais sobre controle de do risco em encostas foram realizados nos anos seguintes. Estas ações constituíram-se como marcos relevantes para a gestão do risco de desastre no Brasil, e finalmente, esta temática passava a estar presente nas agendas municipais.

Em 2008 e 2010, grandes desastres "naturais" foram registrados em Santa Catarina, Alagoas, Pernambuco (Freitas et al., 2014), mas foi a partir de janeiro de 2011, após o desastre na região Serrana do Rio de Janeiro, que o governo federal intensificou suas ações, principalmente visando a prevenção.

\begin{tabular}{c|c|c|c|c|c}
\hline (C) Terrae Didat. & Campinas, SP & v.16 & $1-12$ & $\mathrm{e} 020035$ & 2020 \\
\hline
\end{tabular}




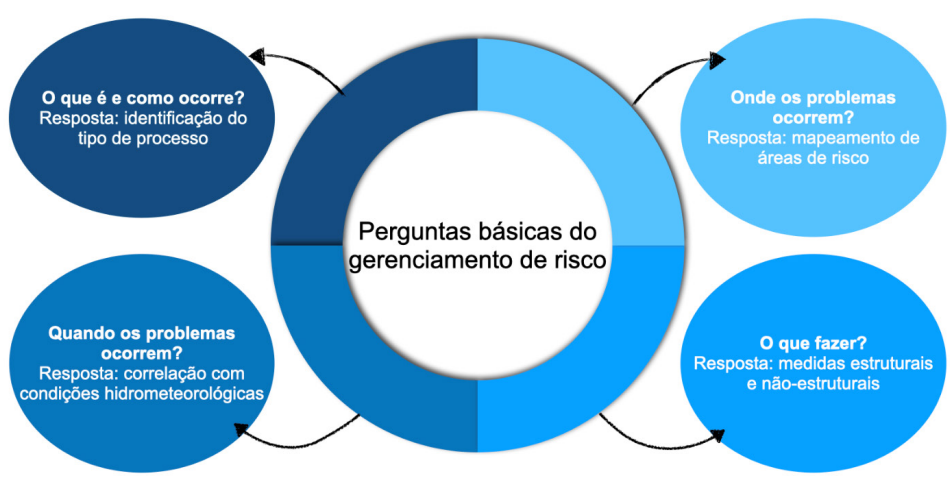

Figura 1. Diagrama das questões essenciais para o gerenciamento de risco. Fonte: (SGB-CPRM, 2017)

Assim, em 2011 o SGB-CPRM passou a integrar o Plano Nacional de Gestão de Riscos e Resposta a Desastres Naturais (PNGRD), a partir de quando foram então iniciadas as ações de setorização emergencial de áreas de risco geológico com abrangência nacional. Desde então, a parceria do SGB-CPRM com as diversas coordenações e secretarias estaduais de Defesa Civil, Cenad e a Cepdec-SP, levou a um grande número de solicitações para cursos de capacitação dos técnicos de defesa civil na identificação de processos geológicos deflagradores destes acidentes.

Ainda no âmbito do PNGRD, destaca-se a criação do Cemaden, em julho de 2011. O desastre da região Serrana do Rio de Janeiro acentuou a premência de um sistema de monitoramento e alertas, focado para o risco de desastre de deslizamentos, inundações e enxurradas, que se constituem como aqueles que mais causam vítimas no país (Ceped, 2013). Deste modo, em caráter inovador no país, um órgão federal passou a reunir competências científicas, tecnológicas e de desenvolvimento para realizar o monitoramento ininterrupto das condições deflagradoras de desastres (Cemaden, 2020).

Ao mesmo tempo em que estas ações ocorreram, em julho de 2014, o Cemaden e a Cepdec-SP por meio de um Acordo de Cooperação Técnica (ACT) passaram a atuar conjuntamente tendo por objetivo:

(...) propiciar a atuação conjunta do Cemaden e da Cepdec para a obtenção de dados ambientais (pluviométricos, hidrológicos, geotécnicos ou outros) disponíveis em tempo real, além do compartilhamento de informações, com a finalidade de propiciar ações de monitoramento e alerta de desastres de esforços e o desenvolvimento de atividades e pesquisas na área de desastres naturais [...] (Diário Oficial da União, 2014, p. 13)
Em cumprimento ao estabelecido no plano de trabalho do ACT, no primeiro semestre de 2016, SGB-CPRM, Cemaden e Cepdec-SP iniciaram a concepção de um curso capaz de integrar as informações e o conhecimento, intitulado "Práticas de Prevenção e Gerenciamento de Riscos de Desastres Naturais (PPGRDN)". Anteriormente, o SGB-CPRM em atendimento à demanda da Cepdec-SP, já ministrava curso voltado para a setorização de áreas de risco de deslizamentos e inundações.

Os partícipes envolvidos concordaram que a duração do curso seguiria ao que já era praticado nos cursos ministrados pelo SGB-CPRM, ou seja, cinco dias úteis, abrangendo conteúdo teórico e prática de campo. Em comum acordo, o curso foi programado para o mês de setembro, período menos chuvoso do ano, o que viabilizaria a participação dos técnicos sem comprometimento de suas atividades locais. Novos conteúdos foram agregados ao curso, de modo inédito, como monitoramento e alertas e planos de contingência, expertises do Cemaden e Cepdec-SP. O propósito foi mostrar a integração de tais áreas, aplicadas ao gerenciamento de risco no nível local.

Durante as reuniões preparatórias foi acertado entre as instituições organizadoras, que dada à densidade de conteúdos que deveria ser abordada ao longo do curso, seria necessário o desenvolvimento de material complementar de cada tema. Uma preocupação inicial foi introduzir conteúdos técnicos, para um público de formação e experiências bastante heterogêneas. Assim, um glossário conjunto foi elaborado e encaminhado aos participantes na semana que antecedeu o curso. Cada instituição também se responsabilizou em elaborar apostila abordando os principais tópicos do curso. Todo material foi disponibilizado aos inscritos em uma pasta online, de modo a evitar o uso de impressão.

\section{$1^{a}$ Edição do curso "Práticas de Prevenção", primavera de 2016}

O processo de inscrição online foi coordenado pela Cepdec-SP e a seleção dos participantes foi realizada em parceria com o Cemaden. No total, foram recebidas 116 inscrições, dentre as quais foram selecionados 36 técnicos de defesa civil municipal 
e 8 alunos de pós-graduação e professores de ensino superior, totalizando assim, 44 participantes de 20 municípios: Atibaia, Caraguatatuba, Campinas, Cajati, Canas, Embu das Artes, Jacareí, Lorena, Marília, Ourinhos, Poá, Pirassununga, Perus Ribeirão Pires, São Paulo, São José dos Campos, Santo André, São Caetano do Sul, Tremembé e Taubaté.

A primeira edição da capacitação foi realizada no período de 12 a 16 de setembro de 2016, no Parque Tecnológico de São José dos Campos, onde ficam as instalações do Cemaden, conforme programação apresentada na Tabela 1.

O primeiro dia do curso foi destinado à apresentação do conteúdo, dinâmica, introdução ao que é o gerenciamento de áreas de risco, e depois enfoque aos tipos de processos existentes e roteiro metodológico de mapeamento, visando à identificação em campo. Estes conteúdos foram apresentados pelo SBG-CPRM, pelos técnicos Andrea F. Lazaretti, Luiz Fernando dos Santos e Gilberto Lima.

O segundo dia tratou sobre o tema monitoramento e alertas, cujo conteúdo foi ministrado pelo Cemaden. Pela manhã, foi realizada a introdução sobre o sistema de monitoramento no Brasil e os recursos que o Centro utiliza para o desenvolvimento de suas atividades. Dentre as ferramentas apresentadas, destacam-se desde o uso de dados observacionais (plataformas de coletas de dados pluviométricos e hidrológicos) e não observacionais (radares e imagens de satélites meteorológicos) até a interpretação dos modelos de previsão do tempo. Como forma de nivelar o conhecimento, foram

Tabela 1. Programação do Curso de Práticas de Prevenção e Gerenciamento de Riscos de Desastres Naturais

\begin{tabular}{|c|c|c|c|c|c|}
\hline Horário & Dia 1 & Dia 2 & Dia 3 & Dia 4 & Dia 5 \\
\hline $\begin{array}{l}08: 00- \\
10: 00 \mathrm{~h}\end{array}$ & $\begin{array}{c}\text { Abertura e } \\
\text { introdução ao } \\
\text { gerenciamento de } \\
\text { áreas de risco } \\
\text { Setorização de } \\
\text { áreas de risco de } \\
\text { deslizamentos e de } \\
\text { inundação }\end{array}$ & $\begin{array}{c}\text { Sistema de monitoramento } \\
\text { e alertas } \\
\text { Ferramentas de } \\
\text { apoio à previsão } \\
\text { hidrometeorológica } \\
\text { aplicada a deslizamento e } \\
\text { inundações }\end{array}$ & $\begin{array}{l}\text { Sistema de } \\
\text { Comando em } \\
\text { Operações/ } \\
\text { Sistema de } \\
\text { Comando de } \\
\text { Incidentes }\end{array}$ & \multirow{3}{*}{$\begin{array}{l}\text { Prática de } \\
\text { campo em } \\
\text { áreas de } \\
\text { risco de } \\
\text { deslizamento } \\
\text { (São José dos } \\
\text { Campos) }\end{array}$} & $\begin{array}{c}\text { Finalização } \\
\text { de atividade } \\
\text { prática - } \\
\text { deslizamentos }\end{array}$ \\
\hline $\begin{array}{l}\text { 10:00 - } \\
10: 15 \text { h }\end{array}$ & Intervalo & Intervalo & Intervalo & & Intervalo \\
\hline $\begin{array}{l}10: 15- \\
12: 15 \mathrm{~h}\end{array}$ & $\begin{array}{l}\text { Setorização de } \\
\text { áreas de risco de } \\
\text { deslizamentos } \\
\text { e de inundação } \\
\text { (continuação) }\end{array}$ & $\begin{array}{c}\text { Continuação do tópico } \\
\text { anterior } \\
\text { Uso do mapa interativo do } \\
\text { Cemaden }\end{array}$ & $\begin{array}{c}\text { Sistema de } \\
\text { Comando em } \\
\text { Operações/ } \\
\text { Sistema de } \\
\text { Comando de } \\
\text { Incidentes } \\
\text { (continuação) }\end{array}$ & & $\begin{array}{c}\text { Finalização } \\
\text { de atividade } \\
\text { prática - } \\
\text { inundação }\end{array}$ \\
\hline 12:15 & Almoço & Almoço & Almoço & Almoço & Almoço \\
\hline $\begin{array}{l}13: 15- \\
15: 15 \mathrm{~h}\end{array}$ & $\begin{array}{c}\text { Roteiro } \\
\text { metodológico para } \\
\text { análise de risco } \\
\text { e setorização de } \\
\text { áreas de risco de } \\
\text { deslizamentos e de } \\
\text { inundação }\end{array}$ & $\begin{array}{l}\text { Simulado de emissão de } \\
\text { alertas }\end{array}$ & $\begin{array}{l}\text { Plano de } \\
\text { contingência }\end{array}$ & \multirow{3}{*}{$\begin{array}{l}\text { Prática de } \\
\text { campo em } \\
\text { áreas de risco } \\
\text { de inundação } \\
\text { (São José dos } \\
\text { Campos) }\end{array}$} & $\begin{array}{l}\text { Discussão da } \\
\text { aula prática - } \\
\text { deslizamentos }\end{array}$ \\
\hline $\begin{array}{l}15: 15 \text { - } \\
15: 30 ~ h\end{array}$ & Intervalo & Intervalo & Intervalo & & Intervalo \\
\hline $\begin{array}{l}15: 30- \\
17: 30 \mathrm{~h}\end{array}$ & $\begin{array}{c}\text { Roteiro } \\
\text { metodológico para } \\
\text { análise de risco } \\
\text { e setorização de } \\
\text { áreas de risco de } \\
\text { deslizamentos e de } \\
\text { inundação }\end{array}$ & $\begin{array}{l}\text { Simulado de emissão de } \\
\text { alertas (continuação) }\end{array}$ & $\begin{array}{c}\text { Plano de } \\
\text { contingência }\end{array}$ & & $\begin{array}{l}\text { Discussão da } \\
\text { aula prática - } \\
\text { inundação } \\
\text { Encerramento } \\
\text { do Curso }\end{array}$ \\
\hline $\begin{array}{l}\text { Respon- } \\
\text { sável }\end{array}$ & SGB-CPRM & Cemaden & Cenad/Cepdec & $\begin{array}{c}\text { SGB-CPRM/ } \\
\text { Cemaden/ } \\
\text { Cepdec }\end{array}$ & $\begin{array}{c}\text { SGB-CPRM/ } \\
\text { Cemaden/ } \\
\text { Cepdec }\end{array}$ \\
\hline \multicolumn{2}{|c|}{ (c) Terrae Didat. } & Campinas, SP & v. 16 & $\mathrm{e} 020035$ & 2020 \\
\hline
\end{tabular}


abordados temas tais como o que representa 1 $\mathrm{mm}$ de chuva em dado período, de que forma os dados são coletados pelos pluviômetros, aspectos introdutórios sobre coleta de dados de radares, satélites meteorológicos, assim como das estações hidrológicas. O intuito foi mostrar aos participantes a possibilidade de realizar o monitoramento de seus municípios por meio do Mapa Interativo, disponibilizado pelo Cemaden. Este recurso online está disponível para a consulta pública em tempo real e há possibilidade de se fazer o download de dados pretéritos da rede observacional do Cemaden.

Nesse módulo ainda foi abordada a forma que os mapeamentos e setorizações de risco, em grande parte elaborados pelo SBG-CPRM, é utilizada na tomada de decisão para o envio de alertas do risco de movimentos gravitacionais de massa e hidrológicos. Na plataforma SALVAR (Sistema de Alerta e Visualização de Áreas de Risco), de uso interno no Cemaden e parceiros, estes mapas e setorizações estão integrados com demais dados ambientais), permitindo análises, como, por exemplo, o valor de precipitação acumulada num dado período próximo à uma área de risco. Por fim, foi destacada a importância do Cemaden receber as informações das ocorrências por parte das coordenações municipais de defesa civil, visando melhorar a qualidade dos alertas e sua antecipação.

No período vespertino, foi realizada uma atividade prática em grupos, de simulado de emissão de um alerta de risco hidrológico e um alerta de risco de movimentos de massa. Para tanto, os grupos deveriam identificar o momento de abertura, alteração de nível e cessar de cada alerta. Ao final, as equipes apresentaram e explanaram sobre as suas escolhas (Fig. 2A), com base no que havia sido ensinado anteriormente, promovendo a discussão com os especialistas em monitoramento do Cemaden, que apresentou a linha do tempo de alertas e ocorrências nos respectivos períodos. As atividades foram conduzidas pelos especialistas em meteorologia, Diego O. de Souza e Caroline E. F. Mourão; em desastres naturais, Maria Cristina Lourenço; em Hidrologia, Graziela B. Scofield; e em Geociências, Carla C. Prieto.

A Cepdec-SP e o Cenad foram responsáveis pelo terceiro dia do curso, destinado às ações práticas de defesa civil, além de mostrar a aplicabilidade, de modo padronizado, de ferramentas de gerenciamento de incidentes, emergências e desastres, além do funcionamento do Incident Command System (Oliveira, 2009). Ainda foi evidenciada a adoção de uma estrutura organizacional integrada, com o objetivo de suprir as complexidades e demandas, extrapolando barreiras jurisdicionais.

Os temas de sistema de comando de operações e planos de contingência foram ilustrados a partir do exemplo de rompimento de barragem de rejeitos ocorrido em Mariana, MG. A discussão despertou grande interesse dos participantes. Ainda foi apresentado o S2iD (Sistema Integrado de Informações
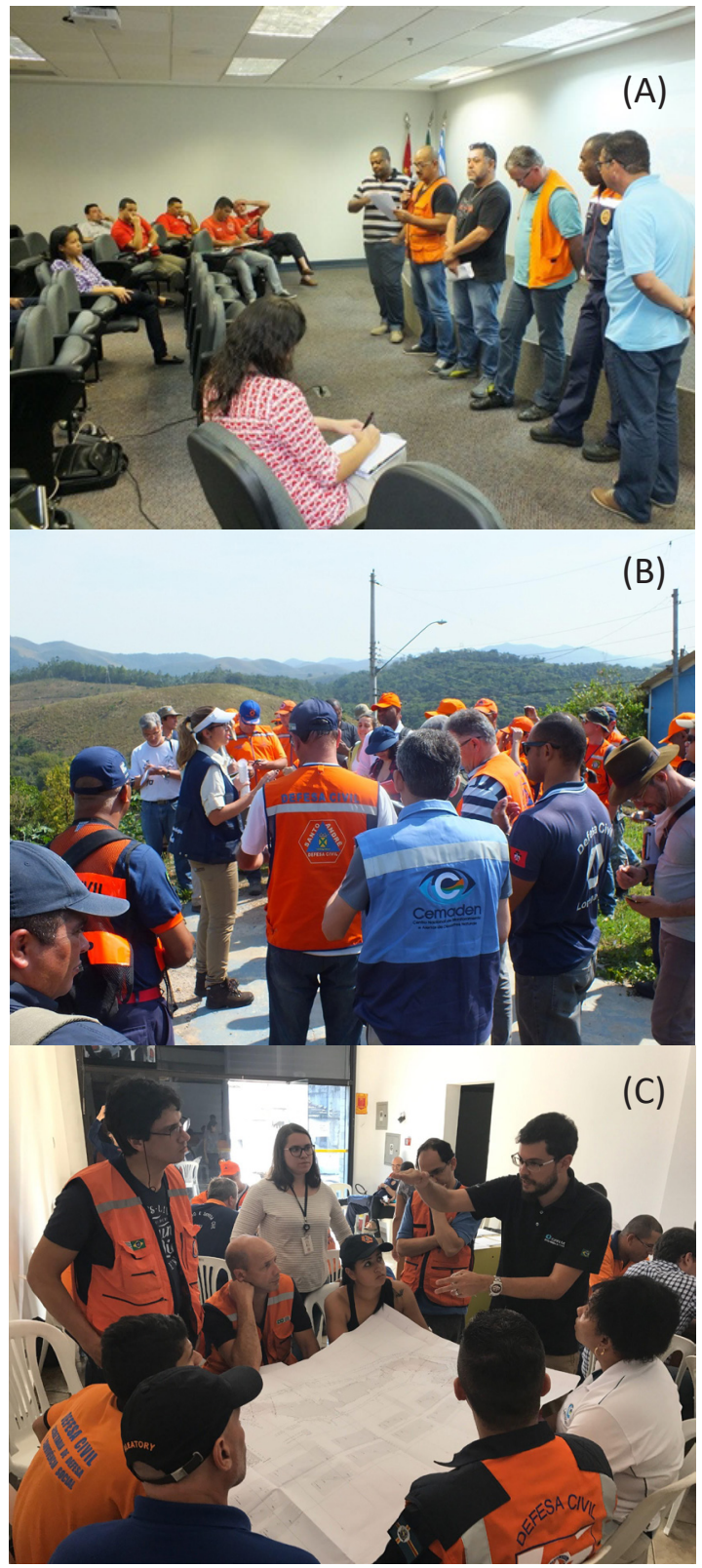

Figura 2. (A) Apresentação dos grupos sobre a linha do tempo dos alertas-simulados. (B) Atividade de campo no Mirante do Buquirinha, em São José dos Campos. (C) Discussão em grupos para delimitação dos setores de risco. Fontes: Fotos A e B: Almeida, 2016; Foto C: Saito, 2017

\begin{tabular}{c|c|c|c|c|c}
\hline (C) Terrae Didat. & Campinas, SP & v.16 & $1-12$ & $\mathrm{e} 020035$ & 2020 \\
\hline
\end{tabular}


de Desastres), bem como instruções básicas para seu preenchimento. Este sistema é destinado a informatizar o processo de transferência de recursos federais para Estados e Municípios afetados por desastres, conforme estabelecido na Portaria MI n ${ }^{\circ} 526$, de 6 de setembro de 2012 (Ministério da Integração Nacional, 2017). Este módulo foi ministrado pelo Cap. Eduardo C. Fernandes Filho, da Cepdec-SP, e Rafael Machado e Cesar da Silva Santana, do Cenad.

Como parte da programação do curso, foi realizado um trabalho de campo no dia 15 de setembro. O objetivo era identificar as principais feições dos tipos de deslizamentos e indícios de inundações, além de familiarizar os participantes com os formulários de campo para este tipo de trabalho. Durante a atividade, em um momento inicial os participantes eram deixados de forma livre para discutirem entre si com alguma ajuda por parte dos instrutores para instigar certas observações. Em um segundo momento, o grupo se reuniu e os instrutores relataram o que estava sendo observado e o porquê. As possíveis ações não estruturais e estruturais que poderiam ser recomendadas na área de estudo também foram debatidas.

A escolha das áreas foi feita em conjunto entre o SGB-CPRM, a Coordenação de Defesa Civil Municipal de São José dos Campos e o Cemaden. Três áreas de risco em São José dos Campos foram selecionadas para a atividade: (i) Mirante do Buquirinha, por apresentar risco de deslizamentos (classificada como risco alto); (ii) Buquirinha, que possui risco de inundação (classificada como risco alto) e (iii) Freitas, uma área com deslizamentos e histórico de óbito, escolhida por ser classificada como risco muito alto (Fig. 2B).

No último dia do curso os participantes foram capacitados a preencher o formulário de campo online (transcrição dos dados de campo) bem como a traçarem os polígonos de áreas de risco utilizando o Google Earth, gerando assim os arquivos . $\mathrm{kml} \mathrm{e}$ .kmz, que são a base dos polígonos utilizados pelo Cemaden na tomada de decisão de envio de alertas.

Ao término das atividades, o encerramento do curso foi feito por representantes do SGB-CPRM, Cemaden, Defesa Civil Municipal de São José dos Campos e Cepdec-SP com a entrega de certificados de participação a todos os envolvidos.

\section{Avaliação dos participantes sobre o curso}

Os participantes avaliaram a primeira versão do curso por meio de formulário semiestruturado. A primeira questão foi voltada aos conteúdos ministrados: $62 \%$ atribuíram conceito ótimo, $38 \%$ como bom, e nenhuma resposta como conceito ruim ou regular. Sobre a duração do curso, $38 \%$ dos participantes consideraram que o tempo não foi suficiente e as principais justificativas estavam relacionadas à densidade dos conteúdos e a necessidade de mais atividade de campo. Quando questionados se estariam aptos a aplicar os conhecimentos em prática, $92 \%$ dos participantes responderam positivamente e $88 \%$ disseram que o conteúdo do curso seria aplicável na rotina de trabalho.

Em relação aos temas que poderiam ser acrescidos em uma nova edição do curso foram apontados: abrigos; como acessar os dados de estações pluviométricas e fluviométricas; capacitação jurídica para defesa civil; mudanças climáticas e ocorrência de desastres; fiscalização em áreas de risco; integração entre geologia, hidrologia e meteorologia no trabalho de defesa civil; padronização de codificação de riscos; R2D2; S2iD; simulado de evacuação em caso de desastres; e vendavais, tornados e micro explosões. Os participantes sugeriram, ainda, a oferta de cursos com maior frequência, a exemplo do PPGRDN, para atender a demanda do público interessado. Ainda foi indicado o uso de outros recursos, como o ensino a distância, para os temas mais introdutórios e conceituais, de modo que o módulo presencial fosse dedicado às atividades de campo, discussão em grupos e mais exercícios práticos.

\section{$2^{a}$ Edição do curso "Práticas de Prevenção", primavera de 2017}

Durante o período de inscrições para o II PPGRDN, 149 pessoas se candidataram para participar do curso, o que evidenciou, mais uma vez, o grande interesse em capacitações dessa temática. Após seleção conduzida pela Cepdec-SP, foram escolhidos 30 profissionais de defesa civil dos municípios de Santa Isabel, São Paulo, Itupeva, Santo André, Ibiúna, Araçariguama, Santana do Parnaíba, Santos, Santa Bárbara d'Oeste, Sumaré, Atibaia, Itu e Guarujá. Houve consenso entre as instituições responsáveis pelo curso em reduzir o número de participantes devido à atividade de campo, que requer atenção dos monitores bem como da logística de deslocamento. Para essa edição do curso, a prioridade foi dada para seleção de participantes com pouco tempo de experiência em defesa civil. Em janeiro de 2017, devido às eleições 
municipais, muitas coordenadorias de defesa civil sofreram alteração de seus quadros técnicos.

A programação do II PPGRDN foi reformulada, diante da experiência vivenciada a partir da primeira edição do curso, bem como dos feedbacks dos participantes. A principal alteração foi a duração do curso, reduzida para 3 dias, diante da dificuldade de muitos se ausentarem de suas atividades por uma semana, dado o quadro reduzido de profissionais de defesa civil nos municípios. Para adequação do tempo, material sobre conceitos básicos foi encaminhado aos participantes com antecedência, de modo a otimizar o conteúdo prático no módulo presencial. O II PGRDN foi realizado de 19 a 21 de setembro de 2017, no Palácio dos Bandeirantes, em São Paulo.

Mais uma vez, o curso foi ministrado por instrutores do Cemaden, Cepdec-SP e SGB-CPRM e contou com módulos teórico e prático. Por incompatibilidade de agenda, essa edição do curso não contou com a participação do Cenad. O trabalho de campo foi realizado com apoio das Subprefeituras de Jaçanã e de Tremembé e da Prefeitura de São Paulo (Fig. 2C).

Os técnicos envolvidos nas aulas práticas e teóricas do II PPGRDN foram:

- SGB-CPRM: Luiz Fernando dos Santos, Gabriel Facuri, Sueli Tomita, Maria Cecília de Medeiros Silveira e Carla Cristina Magalhães de Moraes

- Cemaden: Caroline E. F. Mourão, Regla R. Somoza, Pedro I. Camarinha e Silvia M. Saito

- Cepdec-SP: Eduardo C. Fernandes Filho

\section{Desafios para a prática integrada em GRD em nível local}

A integração entre os diferentes setores envolvidos na prática em GRD sempre foi um princípio buscado no curso PPGRDN, desde sua concepção. Ainda que a abrangência tenha sido restrita à setorização de risco, sistema de alertas e plano de contingência, a observação participante na organização e realização das duas edições do curso possibilitaram a identificação de algumas barreiras para o avanço intersetorial na GRD.

A Lei 12.608/2012 representou um avanço significativo para a GRD no Brasil, em especial por buscar articulação entre os municípios, estados e
União; abordagem sistêmica das ações de prevenção, mitigação, preparação, resposta e recuperação; e integração de políticas setoriais, para promoção do desenvolvimento sustentável (Brasil, 2012). Contudo, por ainda não ter sido regulamentada, resulta que não há meios legais para que os entes possam concretizar todos os pontos supracitados. Por exemplo, a compatibilização de planos diretores, de bacias hidrográficas e de contingência, ocasionando a desnecessária discricionariedade (Carvalho, 2016). Isso remete a implementação na escala municipal, quando se sabe da fragilidade da capacidade econômica e técnico-administrativa no nível local (Nogueira et al., 2014)

Ademais, a constante alteração do quadro técnico dos órgãos municipais de defesa civil, eventualmente substituídos ou destituídos de suas funções (Soriano et al., 2017), leva a um constante recomeço a cada nova gestão municipal. Quando os profissionais estão capacitados, empoderados de suas atribuições, mais experientes e muitas vezes desenvolvendo laços de confiança em comunidades em áreas de risco ao longo do tempo, o vínculo é rompido. Assim todos os dados coletados e o trabalho desenvolvido durante um, dois ou quatro anos (uma gestão municipal completa) são perdidos. Além da escassa otimização de recursos humanos e materiais da administração pública, isso acaba por impactar também a credibilidade junto às comunidades.

Do ponto de vista da formação de recursos humanos, outro desafio constatado foi referente ao volume, diversidade e a quantidade de informações dos temas apresentados ao longo dos cursos, por vezes muito densos, para o público não técnico. Diante da heterogeneidade da formação dos técnicos, característica muito própria da defesa civil brasileira, ficou evidente a dificuldade em lidar com os dados ambientais, tanto o entendimento da aquisição, quanto aos usos e resultados esperados. Alguns participantes relataram muitas dúvidas em assimilar valores de precipitação $\mathrm{em} \mathrm{mm} / \mathrm{h}$, medida essencial para limiares críticos para deflagrar deslizamentos ou ações para os planos de contingência. Outras questões foram levantadas durante as atividades do simulado de emissão de alertas, como o tempo de atualização das informações observacionais e não observacionais e o quanto isso influencia na tomada de decisão de envio de alertas e suas alterações de nível. Os participantes expuseram a dificuldade em identificar o momento ideal para abertura e alteração de nível dos alertas, refletindo

\begin{tabular}{l|l} 
(C) Terrae Didat. & Campinas, SP
\end{tabular} 
sobre o impacto que o evento de precipitação pode gerar. Em face ao exposto, nota-se a necessidade de ampliar, sob o aspecto qualitativo e quantitativo, o quadro de profissionais envolvidos em proteção e defesa civil municipal.

\section{Discussão}

As duas edições do curso Práticas de Prevenção e Gerenciamento de Riscos de Desastres Naturais (PPGRDN) mostraram que técnicos de defesa civil municipal aceitam amplamente a proposta de se capacitar, para estar mais bem preparados para trabalhar na prevenção de desastres. O curso descrito, nas duas edições, é exemplo concreto de ação interinstitucional para promover o gerenciamento de risco de desastres em nível local, desde a identificação dos riscos à preparação. Alguns avanços trazidos pelo curso foram: (i) a maior proximidade e a melhor comunicação das instituições dos três níveis - federal, estadual e municipal, favorecendo o fluxo de informações; (ii) a discussão sobre a importância de ação preventiva em nível local e não apenas em resposta a desastres que eventualmente ocorram; (iii) a promoção da autonomia dos municípios para a identificação e monitoramento de riscos de desastres, bem como na formulação de planos de contingência, em conformidade com a Lei 12608/2012.

Uma dificuldade remanescente é compatibilizar a carga horária com os conteúdos temáticos e a disponibilidade de os participantes se ausentarem dos municípios. A redução do tempo em $50 \%$ na segunda edição do curso, por demanda dos próprios participantes, acabou levando à condensação ou supressão de alguns conteúdos. Por outro lado, a ampla manifestação dos participantes do curso PPGRDN evidencia a necessidade de incorporar outros temas, ou oferecer novos cursos, por macrorregiões dos estados, que proporcionem a formação dos técnicos municipais de defesa civil. Como alternativa para melhor preparação dos participantes, os instrutores consideraram que vídeo-aulas seriam válidas para apresentação de conteúdos teóricos, e o enfoque para a parte prática seria realizado no encontro presencial. O impedimento ficou por conta das verbas para a produção de um material de qualidade e disponibilidade das equipes que realizam o curso, além de outras atividades nas respectivas instituições. Ademais, o ideal seria a dedicação de um grupo permanente, focado em educação em Geociências e redução do risco de desastre, formado por profissionais das instituições envolvidas e demais parceiras. $\mathrm{O}$ apoio financeiro seria essencial para a produção de materiais didáticos, propiciando, assim, a ampliação da rede de técnicos capacitados.

Outro ponto a se destacar é a vulnerabilidade institucional da defesa civil municipal, em especial devido à constante alteração de seu quadro técnico, dificultando que profissionais preparados possam atuar preventivamente em seus municípios em ação contínua, duradoura e com pleno engajamento das comunidades.

\section{Considerações finais}

O curso Práticas de Prevenção e Gerenciamento de Riscos de Desastres Naturais evidenciou aos técnicos de proteção e defesa civil a equidade e integração das áreas envolvidas, isto é, setorização de risco, sistema de alertas e planos de contingência. Ao longo das duas edições do curso, foi constatada a dificuldade de alguns participantes em compreender o uso e aplicação de dados ambientais, o que mostra que pode ser necessária uma formação complementar prévia. Por outro lado, o curso também contou com participantes qualificados ou com vasta experiência em defesa civil. Assim, os próximos cursos serão organizados de modo a atender estes diferentes perfis de profissionais.

Novas edições do curso podem ser realizadas, diante da existência de um acordo de cooperação técnica entre Cemaden e Cepdec-SP. Cabe destacar que o SGB-CPRM mantém cooperação com as duas instituições supracitadas, possibilitando o trabalho conjunto. Espera-se que a presente proposta possa contribuir para colocar em prática as várias diretrizes dispostas pela Lei 12.608/2012, com foco na redução de danos humanos e materiais nos municípios.

\section{Agradecimentos}

A equipe envolvida com a organização, formulação e realização do PPGRDN agradece a todos os participantes, que propiciaram rica discussão ao longo dos cursos; à Defesa Civil de São José dos Campos e as Subprefeituras de Jaçanã e de Tremembé, da Prefeitura de São Paulo, pelo apoio às atividades de campo.

\section{Referências}

Alexander, D., Bramati, L., \& Simonetta, M. (2009). Emergency preparedness training and education in lombardy region, italy: Survey of supply and demand. Natu- 
ral Hazards Review. doi: 10.1061/(ASCE)15276988(2009)10:3(77).

Alvalá, R. C. D. S., Mariane Assis Dias, C. D., Saito, S. M., Stenner, C., Franco, C., Amadeu, P., Ribeiro, J., Souza de Moraes Santana, R. A., \& Nobre, C. A. (2019). Mapping characteristics of at-risk population to disasters in the context of Brazilian early warning system. International Journal of Disaster Risk Reduction, 41. doi: 10.1016/j.ijdrr.2019.101326.

Andjelkovic, I. (2001). International Hydrological Programme Guidelines Non-Structural Measures in. Management, 50(50), 89. URL: http://unesdoc. unesco.org/images/0012/001240/124004e.pdf. Acesso 30.01.2020.

Bertone, P., \& Marinho, C. (2013). Plano de Gestão de Riscos e Resposta a Desastres Naturais. Visão do Planejamento. VI Congresso CONSAD de Gestão Pública, 21, 1-24. Brasília. URL: http://www.sgc. goias.gov.br/upload/arquivos/2013-12/gestao-deriscos-e-resposta-a-desastres-naturais.pdf. Acesso 29.01.2020.

Brasil. (2012) Institui a Política Nacional de Proteção e Defesa Civil, PNPDEC; dispõe sobre o Sistema Nacional de Proteção e Defesa Civil, SINPDEC, e o Conselho Nacional de Proteção e Defesa Civil, CONPDEC. Brasília (DF). URL: http://www.planalto.gov.br/ ccivil_03/_Ato2011-2014/2012/Lei/L12608.htm. Acesso 18.01.2020.

Cardona, O. D. A., Carreño, M.-L., Marulanda, M.C., Barbat, A. H., Saito, S. M., \& Marulanda, P. M. F. (2017). Evaluación del Índice de Gestión del Riesgo de Brasil y resultados comparativos para los países de América Latina y el Caribe. In: Marchezini, S. M., Wisner, V., Londe, B., Saito, L. R. (Eds.). (2017). Reduction of vulnerability to disasters: from knowledge to action. São Carlos: RIMA. p. 632. URL: https://preventionroutes.weebly.com/capiacutetuloschapters.html. Acesso 30.01.2020.

Carvalho, C. S., \& Galvão, T. (2006). Prevenção de Riscos de Deslizamentos em Encostas: Guia para Elaboração de Políticas Municipais. Ministério das Cidades; Cities Alliance. URL: http://planodiretor.mprs.mp.br/ arquivos/prevencaoriscos.pdf. Acesso 30.01. 2020

Carvalho, D. W. (2016). Os Planos Diretores de Bacia Hidrográfica e a irradiação de efeitos sobre instrumentos de ordenação territorial. Revista de Direito Da Cidade. doi: 10.12957/rdc.2016.23805.

Centro Nacional de Monitoramento e Alertas de Desastres Naturais [Cemaden]. (2020). Histórico da criação do Cemaden. São José dos Campos: Cemaden. URL: https://www.cemaden.gov.br/historico-dacriacao-do-cemaden. Acesso 29.01. 2020.

Centro Universitário de Pesquisa e Estudos sobre Desastres (Ceped). (2014). In: Furtado, J. R. (Ed.). (2014). Gestão de desastres e ações de recuperação. Módulo III. Florianópolis: Universidade Federal de Santa Catarina. https://www.ceped.ufsc.br/wp- content/uploads/2013/02/livro-completo-1-1.pdf. Acesso 27.01. 2020

Centro Universitário de Estudos e Pesquisas sobre Desastres (Ceped). (2013). Atlas Brasileiro de Desastres Naturais 1991 a 2012: volume São Paulo. 2 ed. Florianópolis: Universidade Federal de Santa Catarina. URL: https://s2id.mi.gov.br/paginas/atlas/. Acesso 27.01. 2020 .

Coordenadoria Estadual de Proteção e Defesa Civil [Cepdec-SP]. (2020). Histórico. São Paulo: Cepdec. URL: URL: http://www.defesacivil.sp.gov.br/ historico. Acesso 30.01.2020.

Diário Oficial da União (2014). Acordo de Cooperação Técnica, firmado entre a União, por intermédio do Ministério da Ciência, Tecnologia e Inovação - MCTI, Centro Nacional de Monitoramento e Alertas de Desastres Naturais - CEMADEN, e, de outro, a Casa Militar do Gabinete do Governador do Estado de São Paulo CMIL. N ${ }^{\circ}$ 154, quarta-feira, 13 de agosto de 2014.

Di Gregorio, L. T., Soares, C. A. P., Saito, S. M., Soriano, E., Londe, L. de R., \& Coutinho, M. P. (2013). Proposta para a construção um sistema informatizado para gestão integral de riscos de desastres naturais (sigrid) no cenário brasileiro. São Paulo: Departamento de Geografia, Universidade de São Paulo. doi: 10.7154/rdg.2013.0026.0005.

Freitas, C. M., Silva, D. R. X., de Sena, A. R. M., Silva, E. L., Sales, L. B. F., de Carvalho, M. L., Mazoto, M. L., Barcellos, C., Costa, A. M., Oliveira, M. L. C., \& Corvalán, C. (2014). Desastres naturais e saúde: Uma análise da situação do Brasil. Ciencia e Saude Coletiva, 19(9), 3645-3656. doi: 10.1590/1413-81232014199.00732014.

Global Facility for Disaster Reduction and Recovery [GFDRR] \& World Bank [WB]. (2014). Coping with losses: Options for disaster risk financing in Brazil. In: Toro, J.; Matera, M.; Moura, F. S.; \& Pedroso, F.F. (2014). Global Facility for Disaster Reduction and Recovery. Washington: International Bank for Reconstruction and Development URL: https:/www.gfdrr.org/sites/default/files/ publication/Options-for-Disaster-Risk-Financing-in-Brazil-English.pdf. Acesso 30.01.2020.

Gruntfest, E. (2000). Nonstructural mitigation of flood hazards. Inland Flood Hazards: Human Riparian and Aquatic Communities. Cambridge, UK, Cambridge University Press. p. 394-410. doi: 10.1017/ cbo9780511529412.016.

Kobiyama, M., Checchia, T., Silva, R. V., Schröder, P. H., Grando, Â., \& Reginatto, G. M. P. (2004). Papel da comunidade e da universidade no gerenciamento de desastres naturais. I Simpósio Brasileiro de Desastres Naturais. Florianópolis: Universidade Federal de Santa Catarina. URL: https://www.ceped. ufsc.br/wp-content/uploads/2014/07/papel_da_ comunidade_e_da_uni..pdf. Acesso 29.01.2020.

Kundzewicz, Z. W. (2002). Non-structural Flood Pro- 
tection and Sustainability. Water International, 27(1), 3-13. doi: 10.1080/02508060208686972.

Londe, L. D. R., Soriano, E., \& Coutinho, M. P. (2015). Capacidades das instituições municipais de Proteção e Defesa Civil no Brasil: desafios e perspectivas. São Paulo: Departamento de Geografia, Universidade de São Paulo. doi: 10.11606/rdg. v30i0.98715.

Ministério da Integração Nacional. (2017). Gestão de Riscos: Noções Básicas em Proteção e Defesa Civil e em Gestão de Riscos. Brasília: Ministério da Integração Nacional. URL: https://defesacivil.es.gov.br/Media/defesacivil/Capacitacao/Material Didático/ Módulo I/Gestão de Risco - Livro Base.pdf. Acesso 28.01.2020.

Monteiro, V. L., \& Brito Junior, I. de. (2017). A situação atual de implementação da política nacional de proteção e defesa civil no estado de São Paulo. Revista Percursos. PerCursos, 18(36), 194-213. doi: 10.5965/1984724618362017194.

Nogueira, F. R., Oliveira, V. E. de, \& Canil, K. (2014). Políticas públicas regionais para gestão de riscos: o processo de implementação no $\mathrm{ABC}$, SP. Ambiente \& Sociedade, 17(4), 177-194. doi: 10.1590/1809-4422asoc1100v1742014.

Oliveira, M. De. (2009). Gerenciamento de Desastres Sistema de Comando de Operações. Livro Texto do Projeto Gerenciamento de Desastres. Sistema de Comando de Operações / Marcos de Oliveira, 74. Florianópolis: Ministério da Integração Nacional, Secretaria Nacional de Defesa Civil, Universidade Federal de Santa Catarina, Centro Universitário de Estudos e Pesquisas sobre Desastres. 74p. URL: https:// www.ceped.ufsc.br/wp-content/uploads/2014/08/ PR-257-SCO-Manual-by-Marcos-de-Oliveira. pdf. Acesso 30.01. 2020.

Oliver-Smith, A., Alcántara-Ayala, I., Burton, I., \& Lavell, A. (2017). A construção social do risco de desastres: buscando as causas de fundo. In: Marchezini, S. M., Wisner, V., Londe, B., Saito, L. (Eds.). (2017). Reduction of vulnerability to disasters: from knowledge to actionfrom knowledge to action. São Carlos: RIMA. p. 97-114. URL: https://preventionroutes.weebly.com/capiacutetuloschapters. html. Acesso 07.01. 2020.

Pires, R. R. C., \& Gomide, A. Á. (2016). Governança e capacidades estatais: Uma análise comparativa de programas federais. Revista de Sociologia e Política. doi: 10.1590/1678-987316245806.

Programa das Nações Unidas para o Desenvolvimento [PNUD]. (2014). Diagnóstico e Análise das Necessidades de Formação em Gestão e Risco de Desastres. Brasília: Secretaria Nacional de Proteção e Defesa Civil. URL: https://info.undp.org/docs/pdc/ Documents/R46/Diagnostico\%20e\%20Analisiscompressed.pdf. Acesso 29.01. 2020

Séguin, E. (2012). A Lei de Defesa Civil: algumas considerações. Revista Interdisciplinar de Direito, 9(1), 207. URL: http://revistas.faa.edu.br/index.php/ FDV/article/view/514. Acesso 30.01. 2020

Serviço Geológico Brasileiro. Companhia de Pesquisa de Recursos Minerais [SGB-CPRM]. (2016). Aula 1. Introdução ao Gerenciamento de Áreas de Risco. São José dos Campos: Cemaden, Cepdec, SGBCPRM. Material didático usado no curso Práticas de Prevenção e Gerenciamento de Riscos de Desastres Naturais (material inédito).

Serviço Geológico Brasileiro. Companhia de Pesquisa de Recursos Minerais [SGB-CPRM]. (2020). Missão, Visão, Valores e Princípios. URL: http:// www.cprm.gov.br/publique/Sobre/Missao\%2CVisao\%2C-Valores-e-Principios-19. Acesso 30.01. 2020

Shaw, R. (2012). Chapter 1 Overview of Community-Based Disaster Risk Reduction. In: Shaw, R. (Ed.) (2012). Community-Based Disaster Risk Reduction (Community, Environment and Disaster Risk Management, Vol. 10), Emerald Group Publishing Limited, Bingley, pp. 3-17. DOI: 10.1108/S20407262(2012)0000010007.

Soriano, É., Hoffmann, W. M. A., \& Araujo, C. De. (2017). Gestão do Conhecimento aplicado aos desastres naturais: o caso da Defesa Civil. Em Questão, 23(3), 207. doi: 10.19132/1808-5245233.207-227.

United Nations International Strategy for Disaster Reduction [UNISDR]. (2015). Sendai Framework for Disaster Risk Reduction 2015-2030..URL: https://www.undrr.org/publication/sendai-framework-disaster-risk-reduction-2015-2030 . Acesso 30.01.2020

United Nations Office for Disaster Risk Reduction [UNDRR]. (2017). Terminology. UNDRR. In: UN Office for Disaster Risk Reduction. URL: https:// www.undrr.org/terminology. Acesso 28.01.2020. 